\title{
Repetitive transcranial magnetic stimulation in the treatment of depression
}

Saxby Pridmore, William Pridmore

\section{Background \\ Transcranial magnetic stimulation (TMS)is a treatment for major depressive disorder that is otherwise resistant to treatment. Although world- leading research in TMS has been conducted in Australia, where it has had some availability for two decades, there is limited familiarity with the treatment in the general medical community. Availability, however, is increasing.}

\section{Objective}

The aim of this article is to inform general practitioners of some scientific and practical aspects of TMS treatment.

\section{Discussion}

In TMS, an electromagnetic apparatus is used to generate small electric currents in targeted regions of the cortex. Anaesthesia is not required, patients remain conscious and there are no seizure or memory problems. TMS is a first-line treatment for treatmentresistant depression. Current evidence indicates that TMS-induced remission is associated with normalisation of connectivity in cortical-subcortical networks.
TRANSCRANIAL MAGNETIC STIMULATION (TMS) is a means of non-invasively stimulating the cerebral cortex. It is an application of electromagnetism, and has a place in diagnostic neurophysiology and the treatment of some neurological and psychiatric disorders.

Here, we describe TMS as useful in the treatment of major depressive disorder (MDD) that is otherwise resistant to treatment. It is currently reserved for patients who have failed to respond to at least one course of an antidepressant medication administered at a sufficient dose for a sufficient period - that is, patients with treatment-resistant depression (TRD). ${ }^{1}$ Currently, TMS is used predominantly in adults, and is effective in young and old alike. ${ }^{2}$ This treatment has had limited availability in Australia in academic, private and public practices for two decades.

\section{History and physiology}

Electroconvulsive therapy (ECT) was first performed in Rome in the 1930s. It has the strongest available antidepressant effect, ${ }^{3}$ but the requirement for general anaesthesia and induction of seizure, and the side effects (including temporary memory problems) make it unacceptable or inappropriate for some patients.

TMS became possible in $1985 .{ }^{4}$

It depends on the following. When electricity passes along a wire, a magnetic field simultaneously extends at right angles around the wire.

Relevant principles are illustrated by the transformer - a device in which a coil of wire in one circuit is placed close to a coil of wire in a second circuit. When a current is passed through the first circuit, a current also passes through the second (to which it is not electrically connected) the magnetic field of the first coil reaches the second coil, and produces the electric current in that circuit.

The second structure need not be a metal coil; the magnetic field emanating from a coil will induce a small current in any nearby conductor, including a human brain.

Unlike electricity (in ECT), magnetic fields (such as those of TMS) pass directly through the skull (a non-conductor of electricity), with little loss of energy. In the same way, the magnetic field of a magnet held under a wooden table (also a non-conductor of electricity) easily reaches a paperclip on the tabletop. Thus, TMS-generated cerebral electrical activity is tightly focused and can be localised; there is no seizure, no need for anaesthetic, and no memory problems. ${ }^{5}$

The first TMS machines provided only single pulses, followed by a delay during which electrical charge re-accumulated. However, the development of the thyristor (a solid-state semiconductor device) enabled the building of machines, in the mid-1990s, capable of delivering repetitive TMS (rTMS) pulses at up to $20 \mathrm{~Hz}$. Physiological studies have demonstrated that low-frequency $(<1 \mathrm{~Hz})$ rTMS decreases the activity of neural tissue, ${ }^{6}$ while high-frequency $(>1 \mathrm{~Hz}$ ) TMS increases neural activity. ${ }^{7}$ Neuroimaging studies indicated that in MDD, the left dorsolateral prefrontal cortex (DLPFC) is underactive when compared with the right DLPFC. ${ }^{8}$ Thus, two treatment methods emerged: high frequency to the left DLPFC and low frequency to the right DLPFC.

A successful placebo-controlled, randomised study of high frequency rTMS in TRD was reported in $1997 .{ }^{9}$ Success with low frequency rTMS was reported in $1999 .{ }^{10}$

\section{Imaging}

Contemporary TMS imaging studies have explored connectivity - defined 
as a measure of the extent to which the components (nodes) of a network are connected to one another, and the ease (speed) with which they can 'converse' - of three recently described networks: the default mode network, central executive network and the salience network. All have nodes in the prefrontal cortex (the site of TMS stimulation), and connections to subcortical nodes. Depression is associated with dysfunctional connectivity in these networks, and successful rTMS treatment is associated with normalisation of these abnormalities. ${ }^{11-14}$

\section{Current evidence}

Four recent publications warrant consideration:

1. position statement of the Royal Australian and New Zealand College of Psychiatrists (RANZCP) ${ }^{15}$

2. guidelines prepared by European experts $^{16}$

3. recommendations of the Clinical TMS Society ${ }^{17}$

4. guidelines of the Canadian Network for Mood and Anxiety Treatments. ${ }^{18}$

The Canadian authors ${ }^{18}$ found that rTMS (both high and low frequency) is suitable as first-line treatment for treatmentresistant MDD, grading the evidence as 'Level I'. The European authors ${ }^{16}$ gave rTMS (both high and low frequency) a 'Level A recommendation' and reported 'definite efficacy'. The Clinical TMS Society ${ }^{17}$ reached similar conclusions, and the RANZCP ${ }^{15}$ (using earlier information) found 'robust evidence' of efficacy in MDD.

The above groups found that rTMS is a less effective antidepressant than ECT, although the difference may be slight. ${ }^{16}$ However, rTMS is not complicated by the side effects that may make ECT less acceptable.

\section{Patient selection}

rTMS is currently reserved for adults with well-documented TRD, which can be unipolar or bipolar. Patients frequently continue antidepressants and mood stabilisers when receiving TMS; however, there is need for compliance with local treatment centre protocols. rTMS should be considered before advancing to ECT (when available) in all but the most selfdangerous cases. ${ }^{16}$

The contraindications to treatment vary, depending on local treatment centre protocols. The following is a general guide to contraindications:

- a personal history of seizure; in the case of an individual who does not have epilepsy, but has a strong family history, caution is recommended

- pregnancy

- any condition that may lower the seizure threshold (eg significant past head injury, current alcohol withdrawal)

- intracranial metal objects and devices

- cardiac pacemakers - need to be discussed with a cardiologist.

\section{Treatment}

The patient is seated comfortably and the coil is secured (touching the hair/ scalp) by an adjustable arm (Figure 1). A standard daily stimulation protocol is pulses at $10 \mathrm{~Hz}$ in four-second trains, with 26 seconds of rest between trains, and a total of 75 trains. Thus, 3000 stimuli are delivered in a little over half an hour. A course of treatment is 20 daily sessions over 22 days.

A suitably trained medical practitioner determines the appropriate stimulation details at the first treatment. For subsequent treatments, a trained health professional (usually a nurse) positions the patient and the coil, and remains in the facility throughout the procedure.

Patients who come to rTMS have failed pharmacological therapy and, accordingly, have TRD, a condition in which relapse is a common feature. ${ }^{1}$ Relapse in patients with TRD who are treated with rTMS may be considered as much a function of the difficult-to-manage disorder as a failure of the last treatment. Following rTMS-induced remission, the mean time to relapse is five months, ${ }^{19}$ which is similar to that of ECT.

\section{Adverse effects}

Adverse effects are generally trivial rather than serious and there are no long-term undesirable effects. Magnetic pulses may cause scalp discomfort, which usually becomes less annoying for the patient as the course progresses. Some patients develop headache, which responds to simple analgesia - long-term headaches are not a consequence. Anecdotal evidence suggests patients with bipolar disorder can begin to experience elevated mood, but they settle with treatment pauses. Seizure has been reported; however, the incidence $(\sim 0.01-0.1 \%)$ is less than when commencing an antidepressant, and only slightly greater than the spontaneous incidence in the general population. ${ }^{18}$

\section{Naturalistic study outcomes}

Given that patients treated with rTMS have TRD, improvement will be difficult to achieve. A meta-analysis of naturalistic high-frequency rTMS to the left DLPFC found $25-35 \%$ remission; ${ }^{20}$ another metaanalysis that considered low-frequency rTMS to the right DLPFC found 35\% remission. ${ }^{21}$

\section{Cost-effectiveness}

Cost-effectiveness studies have compared TMS and ECT. Given TMS involves more treatments (approximately 20, compared with approximately six), ECT might be expected to have unanimous advantage. However, the first study, performed in the US, concluded that with wide availability

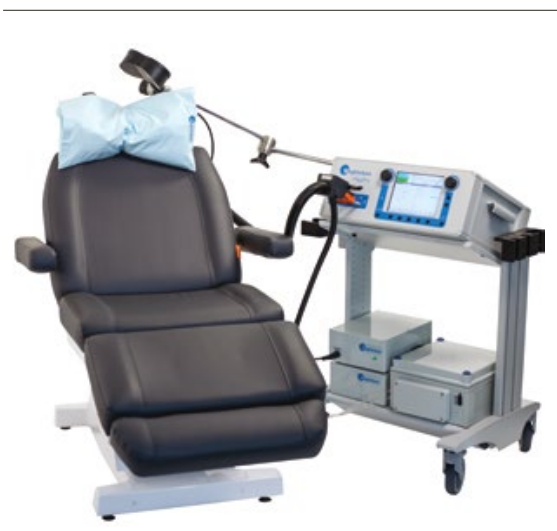

Figure 1. rTMS device with cable and coil secured by mechanical arm

Reproduced with permission from Sonoray Pty Ltd 
TMS would offer an economic advantage and an increase in quality-adjusted life years. ${ }^{22}$ One European study found that, overall, there were no treatment cost differences, ${ }^{23}$ and the most recent study, performed in Singapore, found TMS more cost-effective than ECT over one year. ${ }^{24}$

A recent study in the US reported that for patients newly diagnosed with MDD who have failed a single antidepressant drug trial, there would be cost saving and greater clinical efficacy if the patient was treated thereafter with TMS, for life. ${ }^{25}$ Thus, it can be expected that the future treatment of TRD will involve TMS for at least a proportion of patients.

\section{Other conditions treated}

At this time, we recommend TMS only for the treatment of TRD. Within psychiatry, research continues with low frequency rTMS to the left temporal region as a potential treatment for auditory hallucinations. In neurology, rTMS may have a place in rehabilitation following cerebrovascular accident. ${ }^{26}$ Some benefit has also been claimed in the treatment of tinnitus, Parkinson's disease and chronic pain. ${ }^{27-29}$ TRD is the only condition for which unequivocal evidence is available.

\section{Availability}

rTMS is available in Australia in various academic, private and occasional public settings, but is not widely available. rTMS does not yet have a Medicare Benefits Schedule item number, but should that happen, availability is expected to increase.

\section{Conclusion}

rTMS uses electromagnetism to place small electric currents in localised regions of the cortex. It is effective in the management of TRD, in both placebocontrolled and naturalistic studies. TRD is major treatment challenge and, thus, rTMS is an important medical advance.

rTMS does not cause seizure or the memory problems associated with ECT. The side-effect profile is generally trivial and there are no long-term side effects. rTMS-induced remission is associated with the normalisation of cortical-subcortical connectivity abnormalities. Availability in Australia has been limited; however, services continue to be established and availability is increasing.

\section{Authors}

Saxby Pridmore MBBS, BMedSc, MD, FRANZCP FAFPHM, FAChAM, FFPMAZCP, AM, Professor of Psychiatry, University of Tasmania, Hobart, Tas: Consultant Psychiatrist, Saint Helen's Private Hospital, Hobart, Tas. S.Pridmore@utas.edu.au William Pridmore BA, AdvDipMan, postgraduate student, Australian National Medical School, Gurran, ACT

Competing interests: None.

Provenance and peer review: Not commissioned, externally peer reviewed.

\section{References}

1. Fava M. Diagnosis and definition of treatmentresistant depression. Biol Psychiatry 2003:53(8):649-59.

2. Rostami R, Kazemi R, Nitsche M, Gholipour F, Salehinejad M. Clinical and demographic predictors of response to rTMS treatment in unipolar and bipolar depressive disorders. Clin Neurophysiol 2017;128(10):1961-70.

3. Pridmore S, Bruno R, Turnier-Shea Y, Reid P, Rybak M. Comparison of unlimited numbers of rapid transcranial magnetic stimulation (rTMS) and ECT treatment sessions in major depressive episode. Int J Neuropsychopharmacol 2000;3(2):129-34.

4. Barker AT. An introduction to the basic principles of magnetic nerve stimulation. J Clin Neurophysiol 1991;8(1):26-37.

5. Schulze L, Wheeler S, McAndrews M, Solomon C, Giacobbe P, Downar J. Cognitive safety of dorsomedial prefrontal repetitive transcranial magnetic stimulation in major depression. Eur Neuropsychopharmacol 2016;26(7):1213-26. doi: 10.1016/j.euroneuro.2016.04.004.

6. Chen R, Classen J, Gerloff C, et al. Depression of motor cortex excitability by low-frequency transcranial magnetic stimulation. Neurology 1997;48(5):1398-403.

7. Pascual-Leone A, Valls-Solé J, Wassermann E, Hallett M. Responses to rapid-rate transcranial magnetic stimulation of the human motor cortex. Brain 1994;117(Pt 4):847-58.

8. George MS, Wassermann EM, Williams WA, et al. Daily repetitive transcranial magnetic stimulation (rTMS) improves mood in depression. Neuroreport 1995;6(14):1853-56.

9. George M, Wasserman E, Kimbrell T, et al. Mood improvement following daily left prefrontal repetitive transcranial magnetic stimulation in patients with depression: A placebocontrolled crossover trial. Am J Psychiatry 1997;154(12):1752-56.

10. Klein E, Kreinin I, Chistyakov A, et al. Therapeutic efficacy of right prefrontal slow repetitive transcranial magnetic stimulation in majo depression: A double-blind controlled study. Arch Gen Psychiatry 1999;56(4):315-20.

11. Liston C, Chen A, Zebley B, et al. Default mode network mechanisms of transcranial magnetic stimulation in depression. Biol Psychiatry 2014;76(7):517-26. doi: 10.1016/j. biopsych.2014.01.023.
12. Kang J, Lee $H$, Jhung $K$, et al. Frontostriatal connectivity changes in major depressive disorder after repetitive transcranial magnetic stimulation: A randomized sham-controlled study. J Clin Psychiatry 2016;77(9):e1137-43. doi: 10.4088/ JCP.15m10110.

13. Anderson R, Hoy K, Daskalakis Z, Fitzgerald P. Repetitive transcranial magnetic stimulation for treatment resistant depression: Re-establishing connections. Clin Neurophysiol 2016;127(11):3394405. doi: 10.1016/j.clinph.2016.08.015.

14. Avissar M, Powell F, Ilieva I, et al. Functional connectivity of the left DLPFC to striatum predicts treatment response of depression to TMS. Brain Stimul 2017;10(5):919-25.

15. Royal Australian and New Zealand College of Psychiatrists. Repetitive transcranial magnetic stimulation. Position Statement 79. Melbourne: RANZCP, Practice and Partnerships Committee, 2013. Available at www.ranzcp. org/Files/Resources/College Statements/ Position_Statements/PS-79-PPC-RepetitiveTranscranial-Magnetic-Stimula.aspx [Accessed 24 January 2018]

16. Lefaucheur JP, André-Obadia N, Antal A, et al. Evidence-based guidelines on the therapeutic use of repetitive transcranial magnetic stimulation (rTMS). Clin Neurophysiol 2014;125(11):2150-206. doi: 10.1016/j.clinph.2014.05.021.

17. Perera T, George M, Grammer G, Janicak P, Pascual-Leone A, Wirecki T. The Clinical TMS Society consensus review and treatment recommendations for TMS therapy for major depressive disorder. Brain Stimul 2016;9(3):33646. doi: 10.1016/j.brs.2016.03.010.

18. Milev RV, Giacobbe P, Kennedy SH, et al. Canadian Network for Mood and Anxiety Treatments (CANMAT) 2016 Clinical guidelines for the management of adults with major depressive disorder: Section 4. Neurostimulation treatments. Can J Psychiatry 2016;61(9):561-75. doi: 10.1177/0706743716660033.

19. Demirtas-Tatlidede A, Mechanic-Hamilton D, Press D, et al. An open-label, prospective study of repetitive transcranial magnetic stimulation (rTMS) in the long-term treatment of refractory depression: Reproducibility and duration of the antidepressant effect in medication-free patients. J Clin Psychiatry 2008;69(6):930-34.

20. Carpenter L, Janicak P, Aaronson S, et al. Transcranial magnetic stimulation (TMS) for major depression: A multi-site naturalistic, observational study of acute treatment outcomes in clinical practice. Depress Anxiety 2012;29(7):587-96. doi: 10.1002/da.21969.

21. Berlim M, Van den Eynde F, Daskalakis Z. Clinically meaningful efficacy and acceptability of low-frequency repetitive transcranial magnetic stimulation (rTMS) for treating primary major depression: A meta-analysis of randomized, double-blind and sham-controlled trials. Neuropsychopharmacology 2013;38(4):543-51.

22. Kozel F, George M, Simpson K. Decision analysis of the cost-effectiveness of repetitive transcranial magnetic stimulation versus electroconvulsive therapy for treatment of nonpsychotic severe depression. CNS Spectr 2004;9(6):476-82.

23. Knapp M, Romeo R, Mogg A, et al. Costeffectiveness of transcranial magnetic stimulation vs electroconvulsive therapy for severe depression: A multi-centre randomised controlled trial. J Affect Disord 2008;109(3):273-85. doi: 10.1016/j jad.2008.01.001. 
24. Zhao Y, Tor P, Khoo A, Teng M, Lim B, Mok Y. Cost-effectiveness modelling of repetitive transcranial magnetic stimulation compared to electroconvulsive therapy for treatment-resistant depression in Singapore. Neuromodulation 2017. doi: 10.1111/ner.12723. [Epub ahead of print]

25. Voigt J, Carpenter L, Leuchter A. Cost effectiveness analysis comparing repetitive transcranial magnetic stimulation to antidepressant medications after a first treatment failure for major depressive disorder in newly diagnosed patients - A lifetime analysis. PLoS One 2017;12(10):e0186950. doi: 10.1371/journal. pone.0186950.

26. Askin A, Tosun A, Demirdal S. Effects of lowfrequency repetitive transcranial magnetic stimulation on upper extremity motor recovery and functional outcomes in chronic stroke patients: A randomized controlled trial. Somatosens Mot Res 2017; 34(2):102-07

27. Nurmikko T, Maclver $K$, Bresnahan R, Hird E, Nelson A, Sacco P. Motor cortex reorganization and repetitive transcranial magnetic stimulation for pain - A methodological study. Neuromodulation 2016;19(7):669-78.
28. Thedoroff S, Griest S, Folmer R. Transcranial magnetic stimulus for tinnitus: Using the tinnitus Functional Index to predict benefit in a randomized controlled trial. Trials 2017;18(1):64 doi: 10.1186/s13063-017-1807-9.

29. Cohen O, Rigbi A, Yahalom G, et al. Repetitive deep TMS for Parkinson disease - A 3-month double-blind, randomized controlled study. J Clin Neurophysiol. 2018 Jan 23. doi: 10.1097/ WNP.0000000000000455. [Epub ahead of print]. 